University of Nebraska - Lincoln

DigitalCommons@University of Nebraska - Lincoln

USDA National Wildlife Research Center - Staff Publications
U.S. Department of Agriculture: Animal and Plant Health Inspection Service

$2-2020$

\title{
Effects of Deepwater Horizon oil on feather structure and thermoregulation in gulls: Does rehabilitation work?
}

\author{
Katherine Horak \\ USDA APHIS National Wildlife Research Center, katherine.e.horak@aphis.usda.gov \\ Nicole L. Barrett \\ NWRC, APHIS, USDA \\ Jeremy W. Ellis \\ USDA APHIS Wildlife Services, jeremy.w.ellis@aphis.usda.gov \\ Emma M. Campbell \\ NWRC, APHIS, USDA \\ Nicholas G. Dannemiller \\ Colorado State University \\ Follow this and additional works at: https://digitalcommons.unl.edu/icwdm_usdanwrc

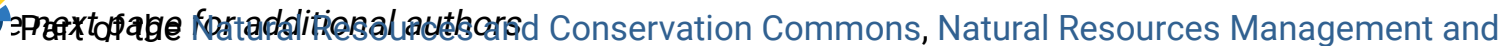 \\ Policy Commons, Other Environmental Sciences Commons, Other Veterinary Medicine Commons, \\ Population Biology Commons, Terrestrial and Aquatic Ecology Commons, Veterinary Infectious Diseases \\ Commons, Veterinary Microbiology and Immunobiology Commons, Veterinary Preventive Medicine, \\ Epidemiology, and Public Health Commons, and the Zoology Commons
}

Horak, Katherine; Barrett, Nicole L.; Ellis, Jeremy W.; Campbell, Emma M.; Dannemiller, Nicholas G.; and Shriner, Susan A., "Effects of Deepwater Horizon oil on feather structure and thermoregulation in gulls: Does rehabilitation work?" (2020). USDA National Wildlife Research Center - Staff Publications. 2336. https://digitalcommons.unl.edu/icwdm_usdanwrc/2336

This Article is brought to you for free and open access by the U.S. Department of Agriculture: Animal and Plant Health Inspection Service at DigitalCommons@University of Nebraska - Lincoln. It has been accepted for inclusion in USDA National Wildlife Research Center - Staff Publications by an authorized administrator of DigitalCommons@University of Nebraska - Lincoln. 


\section{Authors}

Katherine Horak, Nicole L. Barrett, Jeremy W. Ellis, Emma M. Campbell, Nicholas G. Dannemiller, and Susan A. Shriner 


\title{
Effects of Deepwater Horizon oil on feather structure and thermoregulation in gulls: Does rehabilitation work?
}

\author{
Katherine E. Horak ${ }^{\mathrm{a}, *}$, Nicole L. Barrett ${ }^{\mathrm{a}}$, Jeremy W. Ellis ${ }^{\mathrm{a}}$, Emma M. Campbell ${ }^{\mathrm{a}}$, \\ Nicholas G. Dannemiller ${ }^{\mathrm{a}, \mathrm{b}}$, Susan A. Shriner ${ }^{\mathrm{a}}$ \\ a National Wildlife Research Center, Animal Plant Health Inspection Service, US Department of Agriculture, 4101 LaPorte Avenue, Fort Collins, CO 80521, USA \\ ${ }^{\mathrm{b}}$ Department of Clinical Sciences, College of Veterinary Medicine and Biomedical Sciences, Colorado State University, 1601 Campus Delivery, Fort Collins, CO 80523, USA
}

\section{H I G H L I G H T S}

- Pristine feather condition is critical for birds to control body temperature.

- Bird feathers exposed to oil (e.g., from oil spills) become significantly clumped.

- Feather clumping after moderate levels of oiling is evident for at least a month.

- Washing birds after oil exposure reduces clumping to normal levels within 3 weeks.

- Washed birds have normal external temperatures within a week of washing.

\section{A R T I C L E I N F O}

\section{Article history:}

Received 19 December 2019

Received in revised form 15 February 2020

Accepted 15 February 2020

Available online 18 February 2020

Editor: Damia Barcelo

\section{Keywords:}

Feather microstructure

Oil toxicity

Wildlife rehabilitation

Deepwater horizon

Ring-billed gull

FLIR

Thermoregulation

\section{G R A P H I C A L A B S T R A C T}

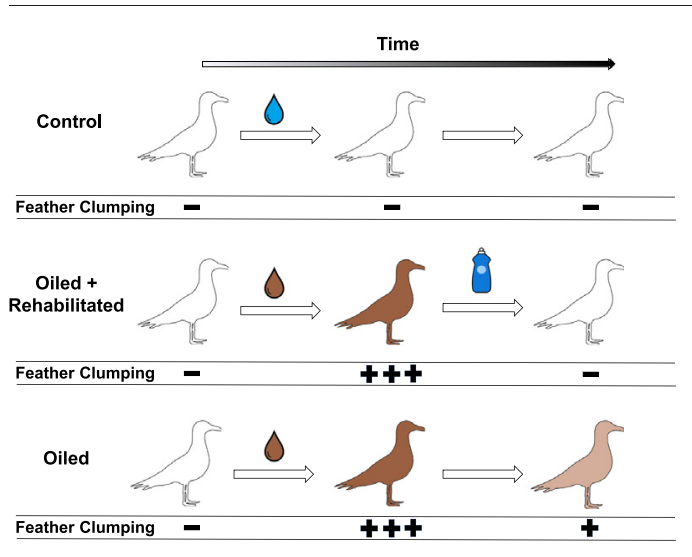

\section{A B S T R A C T}

Impacts of large-scale oil spills on avian species are far-reaching. While media attention often focuses on lethal impacts, sub-lethal effects and the impacts of rehabilitation receive less attention. The objective of our study was to characterize effects of moderate external oiling and subsequent rehabilitation on feather structure and thermoregulation in gulls. We captured 30 wild ring-billed gulls (Larus delawarensis) and randomly assigned each individual to an experimental group: 1) controls, 2) rehabilitated birds (externally oiled, rehabilitated by washing), or 3) oiled birds (externally oiled, not rehabilitated). We externally oiled birds with weathered MC252 Deepwater Horizon oil (water for controls) and collected feathers and thermography imagery (FLIR) approximately weekly for four weeks to investigate feather structure (quantified using a barbule clumping index) and thermoregulatory ability (characterized by internal body temperature and external surface temperature). Post-oiling feather clumping was significantly higher in oiled and rehabilitated birds compared to controls, but steadily declined over time in both groups. However, feather microstructure in rehabilitated birds was indistinguishable from controls within three weeks of washing whereas the feathers of oiled birds were still significantly clumped a month post oiling. Internal body temperatures didn't differ in any of the groups, suggesting birds maintain thermoregulatory homeostasis in spite of moderate external oiling. External temperatures for rehabilitated birds didn't differ from controls within a week of rehabilitation. Overall, rehabilitation procedures were effective and washed birds were in better condition compared to non-rehabilitated, oiled birds. This study provides evidence that the benefits of rehabilitation for moderately oiled birds likely outweigh the costs with regard to

\footnotetext{
* Corresponding author.

E-mail address: katherine.e.horak@usda.gov (K.E. Horak).
} 
feather structure and thermoregulation. While feather preening and time were insufficient to reestablish baseline fine scale feather structure in moderately oiled birds, the significant clumping reduction over time may indicate that rehabilitation of lightly oiled birds may not be necessary and deserves further study.

Published by Elsevier B.V.

\section{Introduction}

Oil spills have dramatic effects on the abiotic and biotic environment ranging from contamination of water, soil, and vegetation to physiologic damage to wildlife from contact with and ingestion of oil, often leading to the deaths of affected animals (i.e. Miller et al. 1978, Horak et al. 2017) . Aquatic birds are often the class of animals most affected by oil spills due do their functional characteristics (Clark, 2001; Munilla et al., 2011; Haney et al., 2014). Dabbling on the water surface, foraging in oiled vegetation, and diving through oil slicks can all result in external oiling of bird species. Because feathers are integral for both thermoregulation and waterproofing, fouling of feathers is a primary cause of morbidity and mortality from oil spills and is of particular concern in cold climates (Hartung, 1967; Leighton, 1993; O'Hara and Morandin, 2010; Dorr et al., 2020) when birds are no longer able to maintain thermoregulation.

While the primary role of feathers for most birds is for flight, feathers also play a significant role in temperature regulation. External oiling can damage feather structure with dramatic effects on birds' ability to thermoregulate. Normally, a thin layer of air is trapped between a bird's skin and its feathers to provide insulation and a barrier between the bird and the environment. If the structural integrity of feathers is compromised, the trapped air and associated body heat can escape, impairing a bird's ability to thermoregulate by widening the temperature differential between the bird's body temperature and the surrounding air and water (Lambert et al., 1982; O'Hara and Morandin, 2010; Rijke and Jesser, 2011; Fritt-Rasmussen et al., 2016). Feathers also play an essential role in waterproofing. The loss of waterproofing that occurs when feather structure is disrupted reduces buoyancy and allows water to permeate the feathers further impairing thermoregulation.

Rescue and rehabilitation of aquatic species is a major component of most oil spill responses and often garners significant media attention. However, the efficacy of such efforts and the long-term survival of rehabilitated birds have been questioned (Sharp, 1996; Estes, 1998; Jessup, 1998; De La Cruz et al., 2013; Henkel and Ziccardi, 2018). The success of oiled bird rehabilitation has been studied in myriad ways, from post-release survival and breeding success studies to evaluation of methods of cleaning (Jenssen and Ekker, 1988; Goldsworthy et al., 2000a; Goldsworthy et al., 2000b; De La Cruz et al., 2013; Finlayson et al., 2018; Golightly et al., 2019). Results from post-rehabilitation survival studies vary and have been found to be influenced not only by the efficacy of washing, but also by the species rehabilitated and the level of oiling, body condition, age class, and environmental temperature at capture (Goldsworthy et al., 2000a; Romero et al., 2018). Because birds spend a significant proportion of their time preening and maintaining their feathers, some oiled birds may have the ability to clean themselves. Therefore, the additional stress of capture, handling, cleaning, and captivity on oiled birds combined with the economic costs of rescue and rehabilitation efforts may outweigh the benefits of rehabilitation (Birkhead et al., 1973; Dixon and Dixon, 1976; Camphuysen, 2011; Duerr, 2013). In most previous studies examining feather microstructure, oil was applied to feathers after they were removed from birds (O'Hara and Morandin, 2010; Morandin and O'Hara, 2014; Bigger et al., 2017; Whitmer et al., 2018; Matcott et al., 2019), but no studies to date have evaluated feather microstructure after applying oil directly to birds to simulate natural exposures. While previous studies all establish significant effects on feather microstructure, particularly from clumping of barbules, none account for the potential mediating effect of time and preening behaviors on feather structure maintenance which may lead to potential recovery in the absence of rehabilitation.

In this study, we experimentally examined the effects of sub-lethal external oiling and rehabilitation on feather microstructure and thermoregulation of ring-billed gulls (Larus delawarensis). The study was conducted in large, outdoor flight pens which allowed birds to perform typical behaviors (preening, flying, swimming) and interact with conspecifics. We had two primary objectives: 1 ) to characterize changes in feather microstructure and thermoregulation over time after external oiling and 2) to assess feather microstructure and thermoregulation after standard rehabilitation procedures.

\section{Material and methods}

All animal procedures were approved by The Institutional Animal Care and Use Committee of the US Department of Agriculture, Animal and Plant Health Inspection Service, National Wildlife Research Center (NWRC; Approval 2806). Birds were collected under Colorado Scientific Collection License 18TRb2433 and Federal permit MB019065. Birds were captured in March 2018. The experiment started on April 29, 2018 (first day of oiling) and continued through May 30, 2018 (31 days).

\subsection{Experimental design}

The experiment evaluated three treatment groups of 10 birds each. Control birds were externally painted with water, rehabilitated birds were externally painted with artifically weathered MC252 Deepwater Horizon oil and then rehabilitated after 8 or 9 days, and oiled birds were externally painted with oil but not rehabilitated during the study period. We collected feathers, thermal images, and body temperatures approximately weekly (days $0,7,15,22$, and 31 with an extra set of thermal images collected on day 2) across a month to assess feather microstructure and thermoregulation. At the end of the study, oiled birds were cleaned and all birds were monitored to ensure they did not exhibit residual effects from the experiment (assessments included behavioral observations, external examination, body weight, internal body temperature, and hematocrit levels). All birds deemed healthy were released near the original capture site or were transferred to another study.

\subsection{Bird capture and husbandry}

Thirty-five ring-billed gulls were trapped in northern Colorado using cannon nets and net guns. Birds were transported to the National Wildlife Research Center (NWRC, Fort Collins, CO USA) in ventilated crates with a transport time of $<30 \mathrm{~min}$ ). Thirty-five birds were captured to ensure at least 30 suitable individuals were available for the experiment (i.e., individuals with no underlying physical conditions such as injury or disease and/or possible mortality during quarantine). Upon arrival to NWRC, birds were quarantined for a minimum of seven days during which they were examined by the attending veterinarian, weighed, and given a unique leg band for identification. Cloacal temperatures and baseline blood samples were also taken. Birds were group housed by treatment group in netted outdoor flight pens $(18.3 \mathrm{~m} \times 37.8 \mathrm{~m})$ with a grass bottom. Each group was maintained in separate, but adjacent flight pens. Outdoor pens contained artificial pools, heat lamps, loafing sheds, and other platforms for perching and shade. Birds were fed an ad libitum mix of Mazuri Fish Analog diet (50/10 Frozen 5 T8, Purina Mills, St. Louis, MO, USA), raw golden shiner minnows 
(Notemigonus crysoleucas, I.F. Anderson Farms, Inc., Lonoke, AR), and canned mackerel throughout the experimental period. Birds had access to fresh water in artificial pools which were changed every three days or sooner if they became visibly dirty. During the experimental period, birds were observed three times daily (approximately 0700, 1200, and 1700) for signs of distress.

\subsection{External oiling}

We obtained artificially weathered MC252 oil from TDI Brooks International Inc. and B\&B Laboratories (College Station, TX, USA) that had been collected from the 2010 Deepwater Horizon oil spill prior to weathering. The artificially weathered MC252 oil was shipped to the NWRC by Ecochem, Inc. (Tukwila, WA) under chain of custody.

Starting on day 0 of the experiment 20 gulls were externally oiled daily for three consecutive days by applying approximately $7 \mathrm{~mL}$ of artificially weathered MC252 oil to the breast, wing tips, and tail feathers with a paint brush. Ten control birds were handled in a comparable manner and painted with $7 \mathrm{~mL}$ water. This quantity of oil was chosen to mimic the upper threshold of the US Fish and Wildlife Service moderate oiling classification for birds which specifies $21-40 \%$ of external surface oiling (Trustee Shorebird Technical Work Group, 2010). We chose to oil the breast, wing tips, and tail feathers to mimic the areas of a surface feeding bird, like gulls, that would most likely come in contact with a floating oil sheen during an oil spill event.

\subsection{Rehabilitation}

Four of the oiled birds in the rehabilitated group were washed on day 8 according to standard practices for rehabilitating oiled birds and the other six were washed on day 9 . We did not rehabilitate all birds in this group on the same day to ensure we had adequate personnel for monitoring. We rehabilitated birds on day 8 or 9 day post exposure to simulate a delay between an oil spill event and the deployment of emergency personnel and prioritization of moderately oiled birds for rehabilitation. Briefly, birds were washed in tubs of warm fresh softened water with 1-2\% detergent (Dawn, Procter \& Gamble, Cincinnati, OH USA). We initially maintained the water at $40-41^{\circ} \mathrm{C}$, but later increased the temperature to a target of $43^{\circ} \mathrm{C}$ due to the higher than average body temperature of the ring-billed gulls (mean cloacal temperature of $42.4^{\circ} \mathrm{C}$ [range 41.4-43.4], Dannemiller et al., 2019). Birds were lightly brushed with soft doll brushes and toothbrushes in the direction of feather growth to prevent damage to the feather structure. One person continuously held a bird's head facing down to prevent soapy water from entering the mouth, eyes, or nares. In deeply oiled or stained areas, a soft toothbrush was dipped in detergent and used to directly clean the feathers. Warmed canola oil $\left(35-38^{\circ} \mathrm{C}\right)$ was sometimes applied to particularly oiled areas prior to washing to remove weathered, tarry oil. In these cases, excess oil was removed using paper towels prior to placing the bird in the first cleaning tub. Bids were moved into consecutive clean soapy tubs once an oil sheen was noticeable and/or the water temperature dropped below $40{ }^{\circ} \mathrm{C}$. Birds were washed until the soapy water remained clean after which birds were rinsed in 1-2 tubs of warm, clear water, followed by a gentle spray rinse to ensure all soap was removed. Birds were thoroughly dried with cloth or paper towels before being gently blown dry with a pet grooming fur dryer (Oster Professional Cage and Table Dryer, Oster, Milwaukee, WI USA) until the feathers were dry to the touch. Birds were then placed in a plastic brooder box outfitted with heat lamps, a warmed towel, and water. Box temperatures were closely monitored and birds were carefully observed for signs of overheating. Birds were kept in the brooder boxes until they began to preen, exhibited signs of activity, and cloacal temperatures were normal. Birds were then released back into the outdoor flight pens and regular monitoring resumed. At the end of the experiment on days 31 and 32 the 10 birds in the oiled group were washed and rehabilitated following the same procedures.

\subsection{Feather structure}

\subsubsection{Feather collection}

Five contour feathers were collected per bird on each sampling day (days $0,7,15,22$, and 31 days post oiling). Feathers were randomly collected from across the entire breast area to avoid concentrating collection in a small area. Feathers were placed in individual manila or wax paper specimen envelopes and kept at room temperature until analysis. Each feather was assigned a random 3-digit number to obscure the treatment group. One of the five feathers from each bird was used to assess microstructure clumping; the other four feathers were used to measure feather oil depth.

\subsubsection{Feather oil depth}

For the oiled birds, we assessed the distance the oil penetrated down the feather vane toward the skin over time. For each of the four feathers, the distance from the tip of the feather (away from the body) to the edge of visible oiling (toward the skin) was measured in millimeters using a ruler. The depth was converted to a proportion by dividing by the length of the rachis (Fig. 3, upper right) and then to a percent by multiplying by 100 . The mean across the 4 feathers was calculated for each bird for each sampling day.

\subsubsection{Feather clumping}

The tip of the remaining feather $(2 \mathrm{~cm})$ was clipped and mounted on a microscope slide with a cover slip attached with cytoseal. The slide was placed on a microscope and two photographs (one of the left side of the rachis and one of the right side) were taken at $100 \times$ magnification which captured approximately $3-5$ barbs in each photograph. Care was taken to center one of the barbs in the image and optimize the focus to that barb. All photos were taken using brightfield illumination with the overhead lights turned off to increase contrast using the image capture software cellSens Entry 1.11, Microscope Olympus BX43 U-LHLEDC (Olympus, Münster, Germany).

To quantify clumping, 10 consecutive barbules from each image were randomly selected from the center of one of the barbs and the number of separated barbules were counted. If none of the barbules were clumped together, the feather was scored as a 10. Likewise, if all of the barbules were stuck together in a single clump the feather was scored as a 1 . If some of the barbules included in the 10 selected for analysis were part of a clump that extended past those 10 barbules, a fraction was added to the clumping index to account for the partial clump. For example, if the last three barbules in a selected group of 10 were part of a clump of 9 ( 3 barbules in the group selected for analysis and 6 barbules outside the selected group), a fraction of 0.33 would be recorded. Therefore, our index values ranged from 1 (maximal clumping) to 10 (no clumping). The mean of the two clumping scores from each of the two images per feather was computed for each bird assessed on each sampling day.

\subsection{Thermography}

On each sampling day, external temperatures of bird breasts and backs were measured using FLIR thermography. Both infrared and visible light images were taken using an infrared camera (ThermaCAM P640, FLIR Systems Inc.). Ambient temperature and relative humidity were recorded before the capture of each image for use in the image analysis software. The camera was placed two meters away from each bird for all images. We held birds by the wings (grasping the joint between the humerus and radius/ulna) and supported them by also holding the head/bill and feet/legs. Care was taken to avoid touching the breast or back to reduce possible effects of handling on surface temperatures. Images were taken in a shaded area to reduce solar radiant heat and in front of a white screen to provide a uniform background. To ensure accurate temperature data was acquired, the camera was focused on the mid breast or back before each photo. Internal body 
temperatures were taken using cloacal thermometers immediately following the acquisition of the photos.

Infrared images were analyzed using FLIR Tools software (FLIR Systems Inc.). Each image had a resolution of $640 \times 480$ pixels, the emissivity was set at 0.95 , and the reflective temperature was set to the ambient temperature. Relative humidity and the $2 \mathrm{~m}$ distance between the camera and bird were also recorded for image analysis. For each image, a $30 \times 30$ pixel measurement box was centered on the bird's breast or back and the minimum, maximum, and mean temperature in the boxed area was calculated.

\subsection{Statistical analysis}

All analyses were conducted in R v. 3.4.3 (R Core Team, 2019). Figures were plotted using ggplot2 (Wickham, 2016).

\subsubsection{Feather oil depth}

Feather oil depth was assessed using basic summary statistics and results were visualized using the R package ggridges (Wilke, 2020).

\subsubsection{Feather clumping}

We ran a repeated measures linear mixed effects model to assess whether barbule clumping varied by treatment group or day post oiling using the R package lme4 (Bates et al., 2015). The model included Clumping as the dependent variable, Treatment group (Control, Rehabilitated, or Oiled), Day post oiling, and an interaction between Treatment and Day post oiling as fixed effects, and ID (individual bird band number) as a random effect. The model did not include data from day 0 since we were interested in estimating clumping effects post oiling. The interaction between treatment group and day post oiling was included based on exploratory data analysis showing a difference in the slopes.

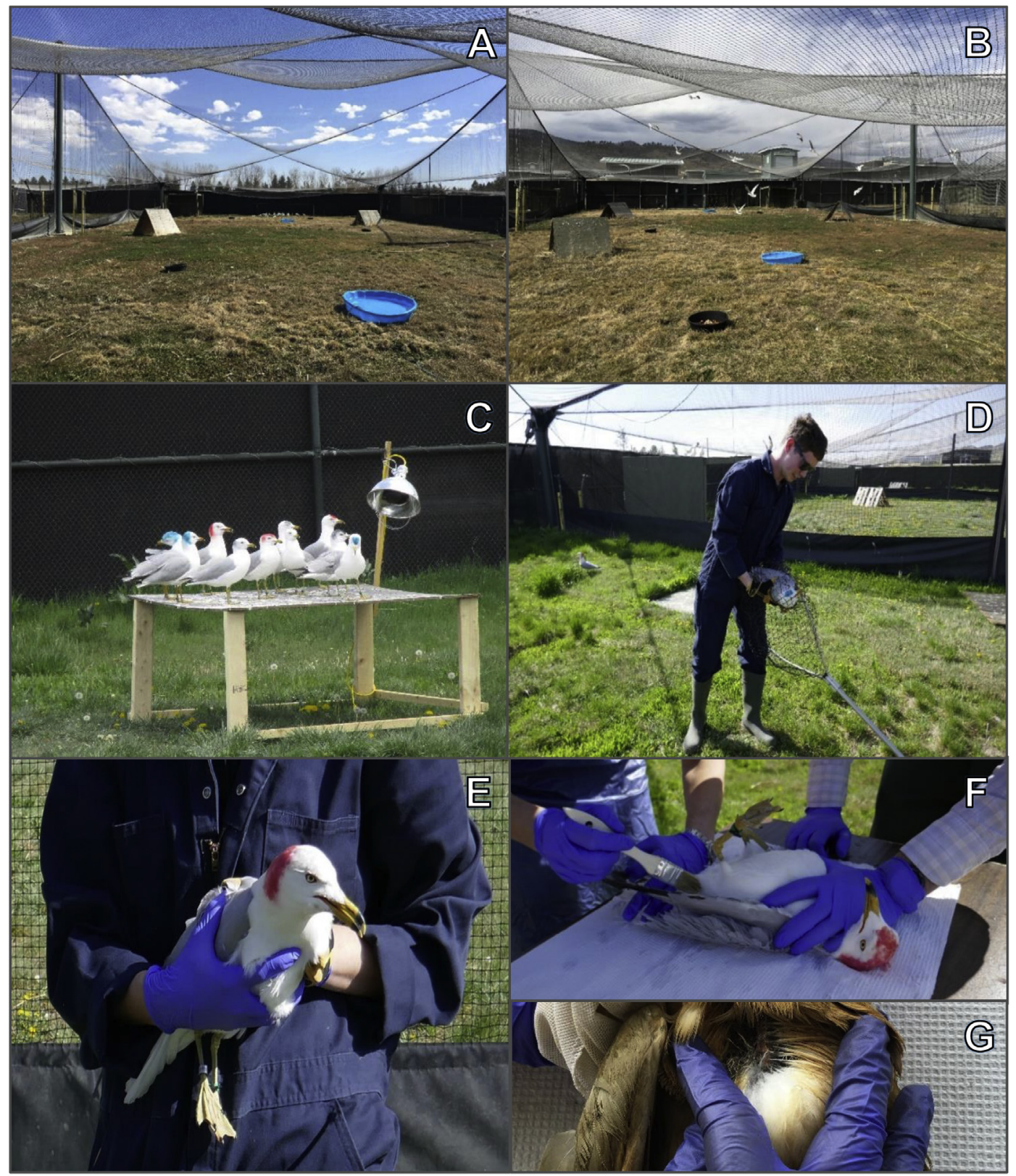

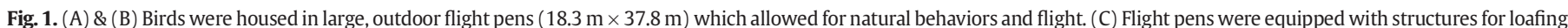

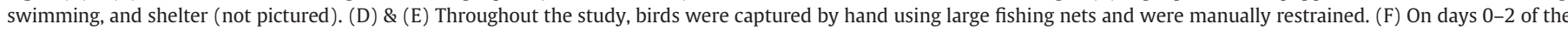

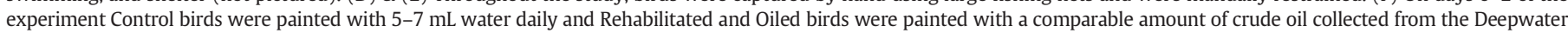
Horizon oil spill. (G) For most Oiled birds, oil never extended to the skin. 


\subsubsection{Thermography}

We modelled the FLIR image data using a simple linear regression model that evaluated the mean FLIR temperature as a function of Treatment group, the Side of the bird evaluated (breast or back), Ambient temperature, Day post oiling, and interactions between Day post oiling with both Treatment and Ambient temperature. We limited the data set to only include days 15-31 because we were interested in evaluating whether rehabilitated birds had external body temperatures different from controls post rehabilitation. We did not evaluate differences between oiled and control birds because we did not have an appropriate emissivity adjustment to correct for the darker color and oil sheen on the oiled birds. A marginal effects plot was created using jtools (Long, 2019).

\section{Results}

\subsection{Feather structure}

\subsubsection{Feather oil depth}

For oiled birds, the depth the externally applied oil moved down the feather toward the skin increased over time post-oiling, increasing the proportion of the feather that was contaminated with oil over time (Figs. 1G, 5). Oil remained visible at the tip of the feathers for the duration of the experiment until rehabilitation. Seven days post-oiling, oil was visible on an average of $70 \%$ of the feather vane length; however, there was considerable variation in the oil distribution across feathers and individuals. This variation decreased over time (Fig. 5). On days 15 and 22 the proportion of the feathers contaminated with oil increased to $83 \%$ and $89 \%$ respectively. By day 31 post-oiling, an average of $91 \%$ of feather vanes were contaminated by oil, but for most feathers examined, oil did not reach the bottom of the feather vane/calamus, leaving a small proportion of the feather that was white and uncontaminated to protect the skin.

\subsubsection{Feather clumping}

Feather clumping was negligible in control birds across all sampling periods, with a slight increase evident on day 7 which was the first sampling post painting (Fig. 4, blue boxes). In contrast, significant clumping was evident seven days post oiling in both rehabilitated (prior to washing) and oiled birds compared to controls ( $\mathrm{p}<0.001$, Table 1, Figs. 3, 4). On day 7 , the mean clumping score $(1=$ maximal clumping, $10=$ no clumping) was 9.6 for control birds, 5.0 for rehabilitated birds, and 4.6 for oiled birds. Both rehabilitated and oiled birds showed a significant, decreasing trend in clumping over time with a significantly steeper

\section{Table 1}

Model estimates for a repeated measures linear mixed effects model of feather barbule clumping by Treatment (Oiled and Rehabilitated compared to Control), Day Post Oiling (DPO), and the interaction between Treatment and DPO. The positive parameter estimates and p-values $<0.001$ for the Oiled and Rehabilitated treatment groups indicate significant feather clumping in those groups compared to Controls. The negative parameter estimates and $\mathrm{p}$-values $<0.001$ for the Trt*DPO interaction terms indicates that feather clumping decreased over time with the Rehabilitated birds showing a significantly faster rate of decline compared to the Oiled birds.

\begin{tabular}{|c|c|c|c|c|c|}
\hline Fixed effects & $\begin{array}{l}\text { Parameter } \\
\text { estimate }\end{array}$ & $\begin{array}{l}\text { Standard } \\
\text { error }\end{array}$ & Df & t-Value & $\begin{array}{l}\operatorname{Pr}(>1 \\
\mathrm{t} \mid)\end{array}$ \\
\hline Intercept & 0.388 & 0.458 & 100.417 & 0.847 & 0.399 \\
\hline $\begin{array}{l}\text { Treatment } \\
\text { Oiled }\end{array}$ & 6.074 & 0.653 & 100.372 & 9.301 & $<0.001$ \\
\hline $\begin{array}{l}\text { Treatment } \\
\text { Rehabilitated }\end{array}$ & 6.405 & 0.665 & 100.417 & 9.629 & $<0.001$ \\
\hline $\begin{array}{l}\text { Day Post Oiling } \\
\text { (DPO) }\end{array}$ & -0.006 & 0.019 & 81.967 & -0.325 & 0.746 \\
\hline $\begin{array}{l}\text { TrtOiled* } \\
\text { DPO }\end{array}$ & -0.117 & 0.028 & 84.678 & -4.142 & $<0.001$ \\
\hline $\begin{array}{l}\text { TrtRehabilitated* } \\
\text { DPO }\end{array}$ & -0.199 & 0.028 & 81.967 & -7.045 & $<0.001$ \\
\hline
\end{tabular}

decline in clumping for rehabilitated birds (post-washing) compared to oiled birds (Fig. 4). On the last day of sampling day 31 post oiling (22-23 days post-washing), feather clumping in the rehabilitated group was not significantly different from the controls, but was still significantly higher in the oiled birds.

\subsection{Thermography}

The weather and ambient outdoor temperature varied greatly during the study. The minimum temperature was $3.1^{\circ} \mathrm{C}$, the maximum was $29.7^{\circ} \mathrm{C}$. The median low temperature was $8.1^{\circ} \mathrm{C}$ and the median high was $23.3^{\circ} \mathrm{C}$. We did not see a difference in cloacal temperatures between control, oiled, or rehabilitated birds at any of the time points. Temperatures ranged from $40.5-44.0^{\circ} \mathrm{C}$ across the experiment (see Dannemiller et al., 2019 for detailed cloacal temperature data). The linear regression model of FLIR image analysis showed that within a week post rehabilitation, external body temperature in rehabilitated birds did not differ from controls ( $p=0.230,10$ birds per group, Figs. 6, 7). From one week post washing to the end of the experiment, there were no significant differences in average external temperatures although rehabilitated birds were slightly warmer on average (Fig. 7). For all birds, the back was significantly cooler than the breast (Fig. 6). We did not acquire accurate FLIR image data for oiled birds as we did not obtain an appropriate emissivity value that adjusted for the dark color and sheen of the oil.

\section{Discussion}

In this study, we experimentally examined the effects of external oiling on thermoregulation and feather structure in live birds housed in large outdoor flight pens which allowed for normal behaviors such as preening and flying. While there are numerous reports of the negative effects of oil on feather structure and barbule clumping (O'Hara and Morandin, 2010; Morandin and O'Hara, 2014; Fritt-Rasmussen et al., 2016; Whitmer et al., 2018; Matcott et al., 2019), studies on live birds have been rare. Similar to previous studies, we found that moderate oiling causes significant barbule clumping. We also examined how rehabilitation impacts barbule clumping and found that feathers from rehabilitated birds were indistinguishable from controls within three weeks of rehabilitation, indicating that rehabilitation does not have long-term detrimental effects to feather microstructure as measured by barbule clumping. We also found a significant decrease in feather clumping over time in oiled birds, but significant clumping was still evident a month post oiling. We assume the decrease in clumping was associated with feather preening and natural wear over time.

The efficacy of rehabilitation and its effect on the survival of oiled birds has been widely studied (Jenssen and Ekker, 1988; Jenssen, 1994; Dannemiller et al., 2019). Many studies show an improvement in body condition and feather structure, but others point to low longterm success following rehabilitation. For example, a study of common murres found a $7 \%$ survival rate of rehabilitated birds at 142 days post-washing (Newman et al., 2004; De La Cruz et al., 2013). Our data show that washing significantly improves feather structure at a much faster rate compared to oiled birds that are not rehabilitated. The plumage of aquatic birds repels water as a function of both feather microstructure and preen oils which are hydrophobic and well developed in aquatic birds compared to land birds (Srinivasan et al., 2014). A potential difference between our study and many of those finding lowered survival rates in rehabilitated birds, may be that our study species is primarily a dabbling bird whereas many studies have focused on diving birds which potentially experience additional stress to their plumage as they dive for prey. In addition, we found that while oil continued to move down the feather toward the skin after the cessation of oil exposure, the oil never reached the skin for the vast majority of feathers evaluated, likely allowing for the maintenance of an intact layer of insulative air. This result is in contrast to a study of external oiling of double- 
crested cormorants using similar methods that found that oil reached the skin of birds (Cunningham et al., 2017).

Similar to previous studies (e.g., Finlayson et al., 2018), we saw high variation in clumping scores for the rehabilitated birds approximately a week after washing. While clumping was statistically similar in rehabilitated and oiled birds a week post rehabilitation, clumping scores were far more variable in the rehabilitated birds compared to the oiled birds with some individuals showing significant improvements. Differences between clumping across individuals may have been related to differences in preening behavior post rehabilitation or were possibly a function of the rehabilitation process. After our sampling a week after rehabilitation, barbule clumping in the rehabilitated birds declined at a much faster rate compared to oiled birds which may indicate that rehabilitation significantly improves feather structure, but that birds should be held for at least two weeks after washing to allow time for preening and reestablishment of normal feather structure. This may be an important consideration for oil-spill responses when birds are released into the wild following rehabilitation efforts.

We were interested in the ability of birds to clean themselves to determine if the stress of the rehabilitation process to the bird is generally warranted as a number of studies discuss the potential ability of oiled birds to clean themselves without human intervention (Birkhead et al., 1973; Phillips, 1974). A study on the survival of gulls found that heavily oil birds survived and managed to clean their own feathers (Camphuysen, 2011). Nevertheless, no experimental evaluation of the ability of oiled birds to reestablish normal feather structure had been conducted. We found that over time, oiled birds were able to clean their feathers and decrease barbule clumping. However, even 31 days post-oiling, contour feathers from gulls that had not been rehabilitated had significantly more barbule clumping than both control and rehabilitated birds. Oiling and feather damage is likely to increase energetic costs (Mathewson et al., 2018) which could lead to reduced survival,
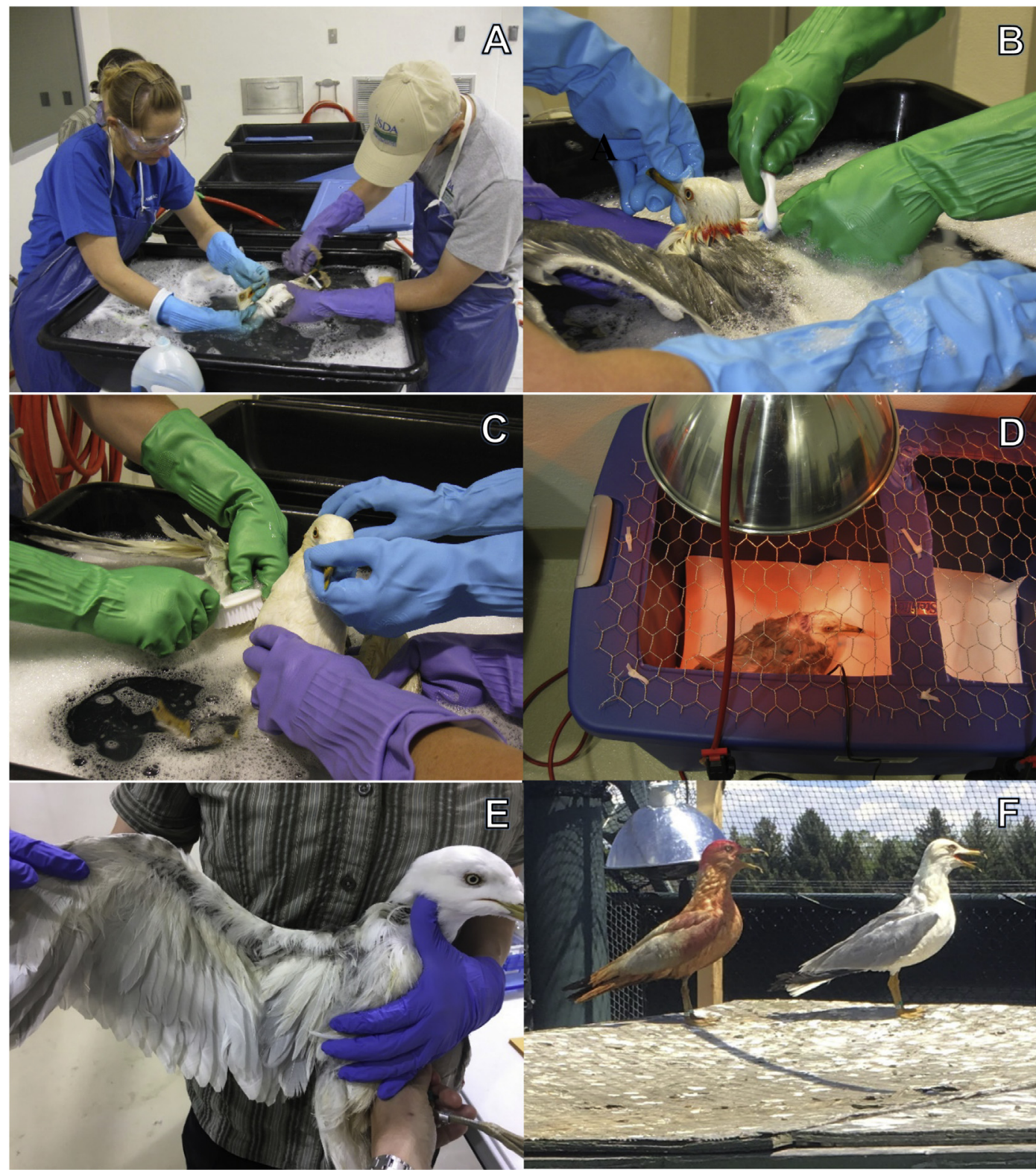

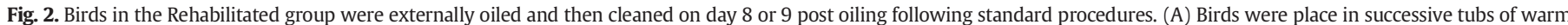

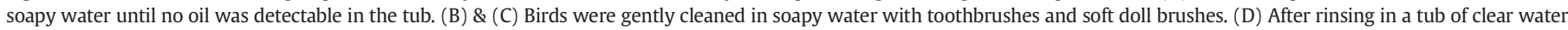

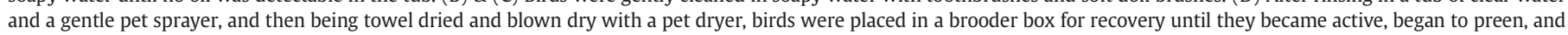
body temperatures were within normal ranges. (E) Some feathers showed minor staining from the oil. (F) A rehabilitated bird next to an oiled bird, yet to be cleaned. 
especially at cold environmental temperatures. Nonetheless, clumping was significantly reduced and birds with only have a small amount of external oiling may be able to preen and clean their feathers without rehabilitation.

Feather structure and thermoregulation are not the only considerations for assessing the cost-benefit trade-offs of rehabilitation. Studies of light oiling have identified significant impacts to flight. A study of light oiling in shorebirds found light oiling increased the energy necessary for flight which could potentially affect migration, time spent foraging for food, survival, and reproduction (Maggini et al., 2017). In another study, light oiling affected the flight patterns of homing pigeons and caused oiled birds to take significantly longer to return to roosts and to spend more time stopping than unoiled birds (Perez et al., 2017). Therefore, based on our findings, rehabilitation of moderate and heavy oiled birds may enhance long-term survival by improving feather structure and concomitant benefits. However, additional research is needed to determine if washing lightly oiled birds is warranted if birds can clean themselves given the stress of rehabilitation and the costs of light oiling on flight, the ability to evade predators, and the potentially increased time spent foraging. Also, the birds in our study had clean food and water ad libitum potentially lessening the impacts associated with oil spills. If birds had to forage for food or only had access to oilcovered food, the negative effects of oil exposure could be exacerbated.

After an oil spill, much effort is dedicated to the identification of oiled birds and the determination of the effects of oil on their thermoregulation. We have previously reported that the internal temperatures of our oiled birds were not significantly different than controls (Dannemiller et al., 2019), indicating that the birds in this study were able to maintain thermoregulatory homeostasis in spite of moderate external oiling. Prior work in cormorants indicates that external oiling can increase heat loss through feather disruption which increases energetic costs (Mathewson et al., 2018). Similar to previous reports, we found

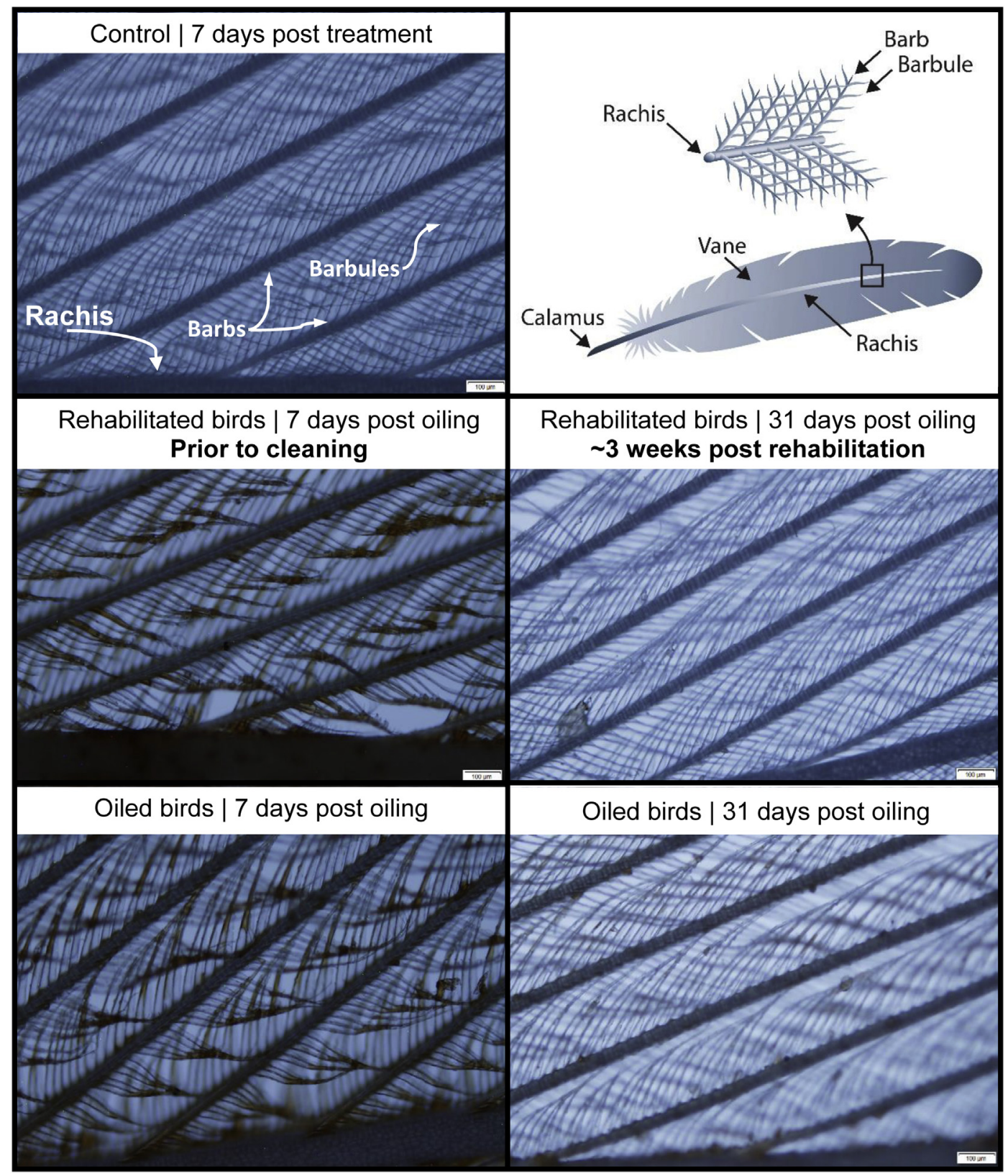

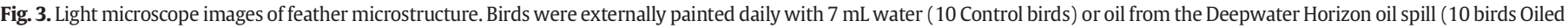

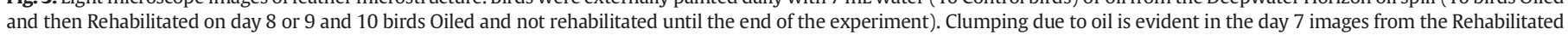
(prior to cleaning) and Oiled birds. All feathers were magnified approximately $100 \times$ using a $10 \times$ objective lens. 


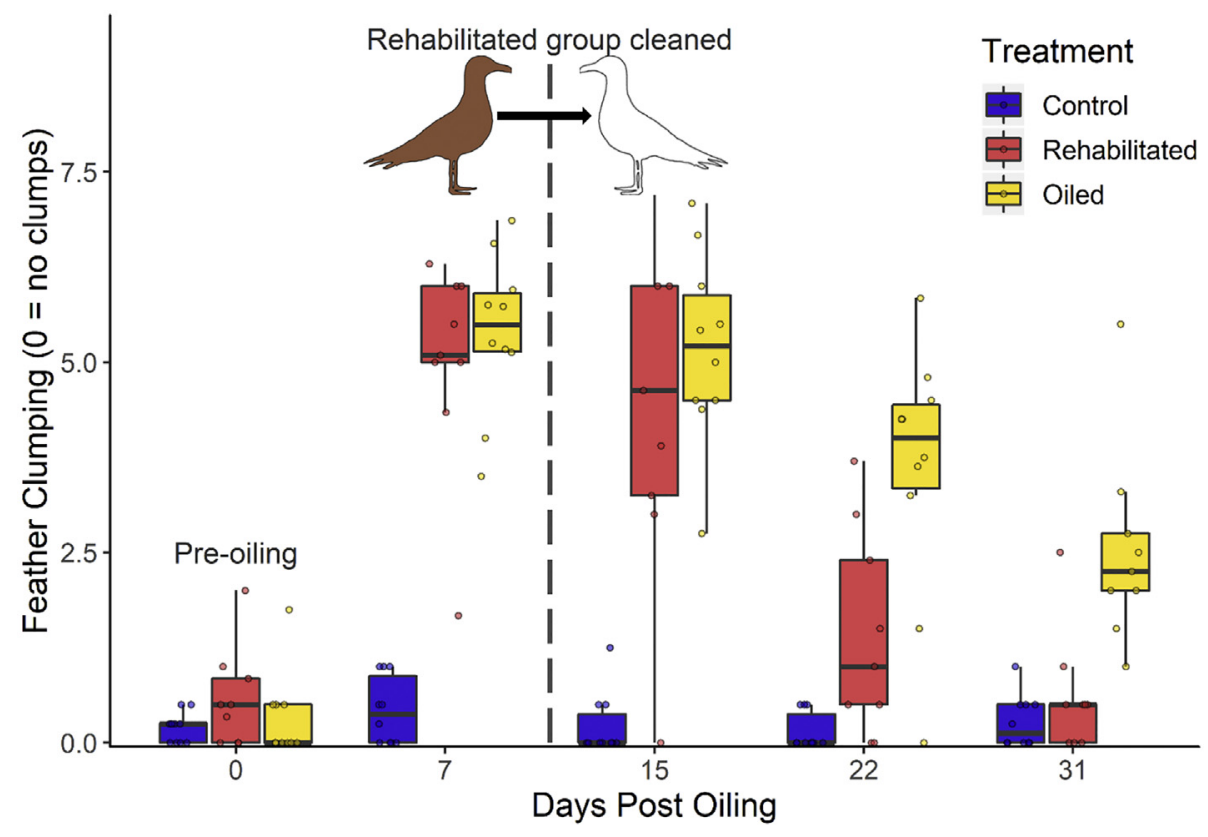

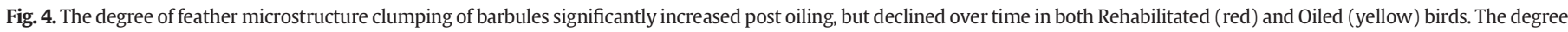

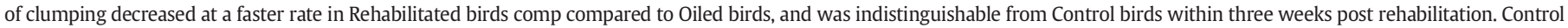
birds (blue) showed minimal clumping at all time points. (For interpretation of the references to color in this figure legend, the reader is referred to the web version of this article.)

variations in surface temperature based on body location with the backs of our control and rehabilitated birds having significantly lower external temperatures than the breasts (McCafferty, 2007; Nevins et al., 2012; Mathewson et al., 2018). In this study, we used birds with white plumage such that oil application changed the color and texture of the feathers (Fig. 2F). Because the determination of external temperatures using FLIR requires an emissivity value specific to the material being imaged, we were not able to apply an appropriate emissivity value for oiled birds since oiling changed feather color and varied across feathers. Changes in surface color have dramatic effects on external temperature measurements made using FLIR thermography. One of the best examples of this is a photo of a zebra in which the stripes cause dramatic variations in temperatures on infrared images (McCafferty, 2007). While FLIR was not useful for determining absolute external temperatures of oiled RGBU in this study, this technology is potentially useful for the

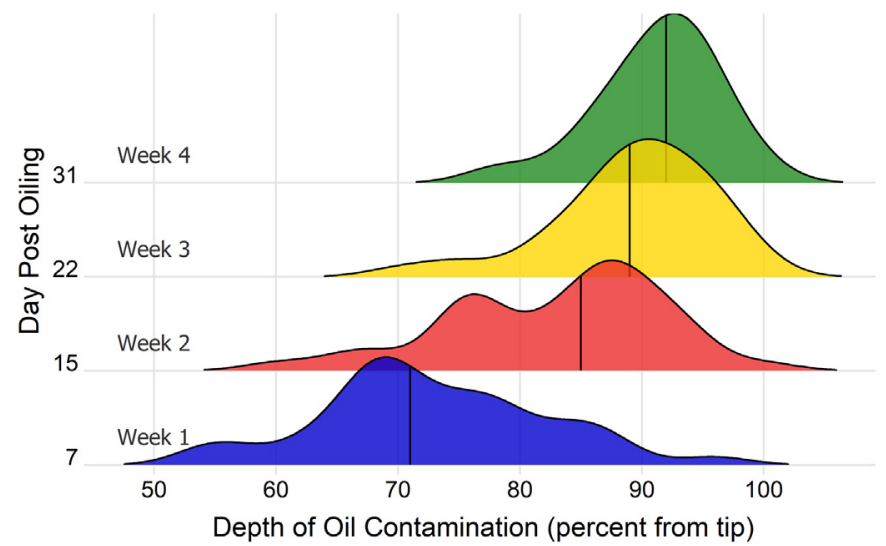

Fig. 5. The proportion of the feather area externally contaminated by oil increased over time, presumably due to a combination of preening and natural spread. For the 10 externally oiled birds, we collected four contour feathers from the breast of each bird and measured how far down the rachis (the primary shaft down the middle of a feather vane) we could visually detect oil as a percentage of the length of the feather vane (see Fig. 2 for a feather schematic). On average, oil contamination covered $70 \%$ of feathers a week post oiling and oil was detectable on $90 \%$ of feather area after 4 weeks. At the end of the 4-week experiment oil did not extend to the bottom of the rachis or reach the skin in a majority of feathers evaluated. identification of oiled areas to inform washing procedures. Studies on a variety of vertebrate species have shown variations in external temperatures associated with changes in the insulative capacity of feathers and fur. Because external oil disrupts feather structure and can cause a loss of insulation, FLIR imagery is well suited to detect oiled areas or identify incompletely washed areas of rehabilitated birds (Jessup, 2010; Nevins et al., 2012) and may be harnessed to improve the efficacy of response efforts.

\section{Conclusions}

Oil spills cause substantial impacts on the environment and avian species. Following oil spills, much effort is devoted to the rehabilitation of oiled wildlife. We were interested in characterizing the effects of external oiling and rehabilitation on feather structure and thermoregulation in ring-billed gulls housed in large outdoor flight pens allowing for normal behaviors such as flying and preening. After moderate levels of external oiling, feather clumping was significantly higher in oiled birds compared to controls. Three weeks post-washing feather structure of rehabilitation birds did not differ from controls. After washing, external temperatures of rehabilitated birds were not significantly different than controls. These findings help inform rehabilitation practices for avian species following external oiling and support the washing of moderately oiled birds. Our results indicate that rehabilitated birds may also benefit from a three-week holding period following washing to allow time for normal preening behaviors to further improve feather structure.

\section{Funding source}

This research was supported by the intramural research program of the US Department of Agriculture, Animal Plant Health Inspection Service.

\section{CRediT authorship contribution statement}

Katherine E. Horak: Conceptualization, Methodology, Investigation, Writing - original draft. Nicole L. Barrett: Methodology, Investigation, Writing - review \& editing. Jeremy W. Ellis: Methodology, Investigation, 


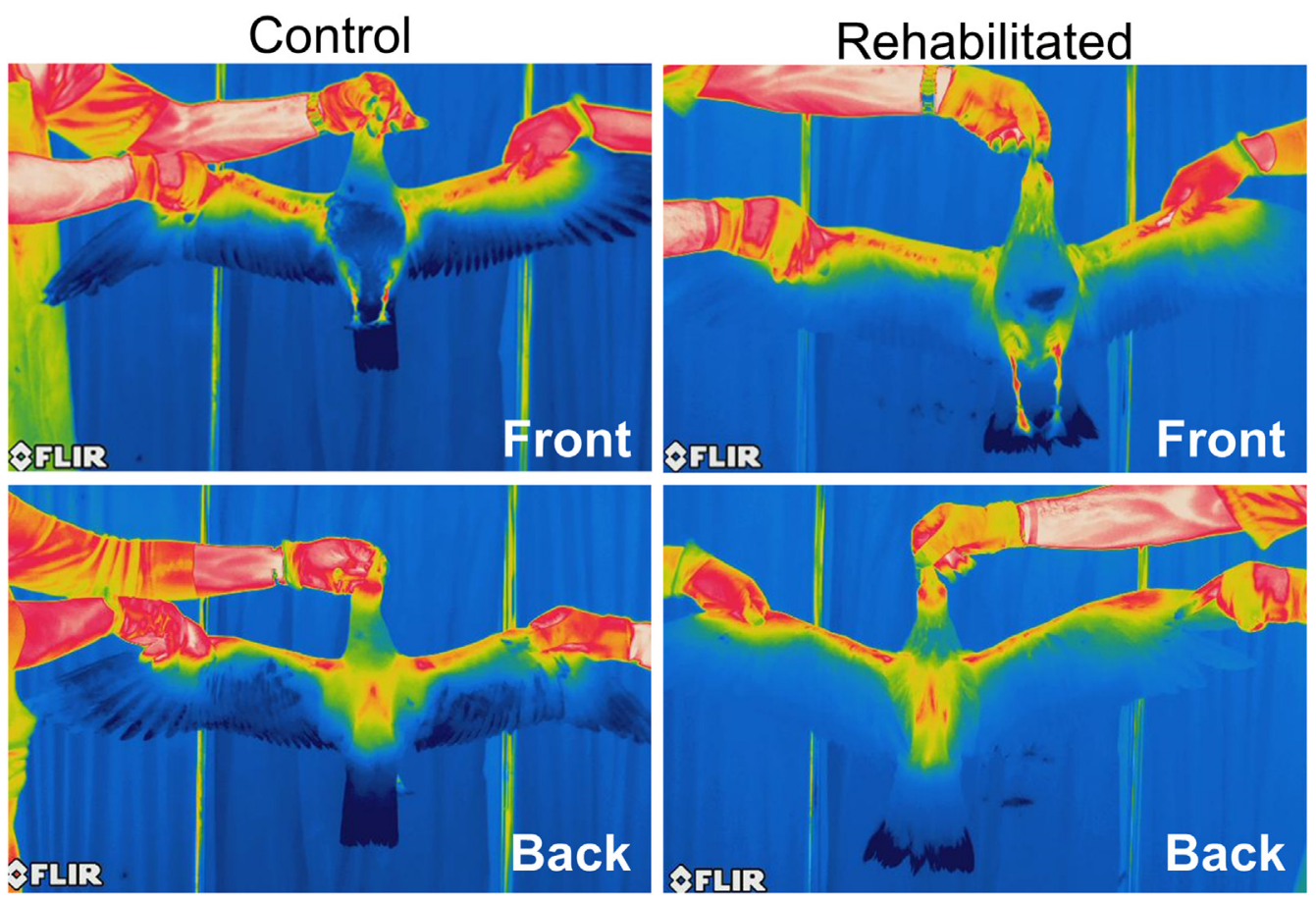

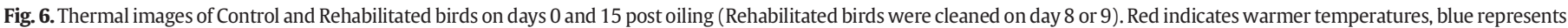

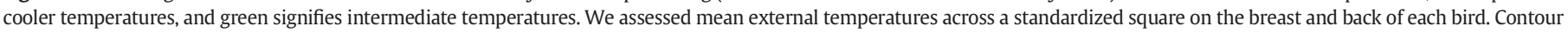

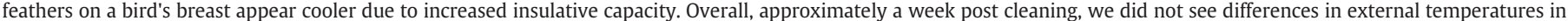
Rehabilitated birds (right) compared to Control birds (left). (For interpretation of the references to color in this figure legend, the reader is referred to the web version of this article.)

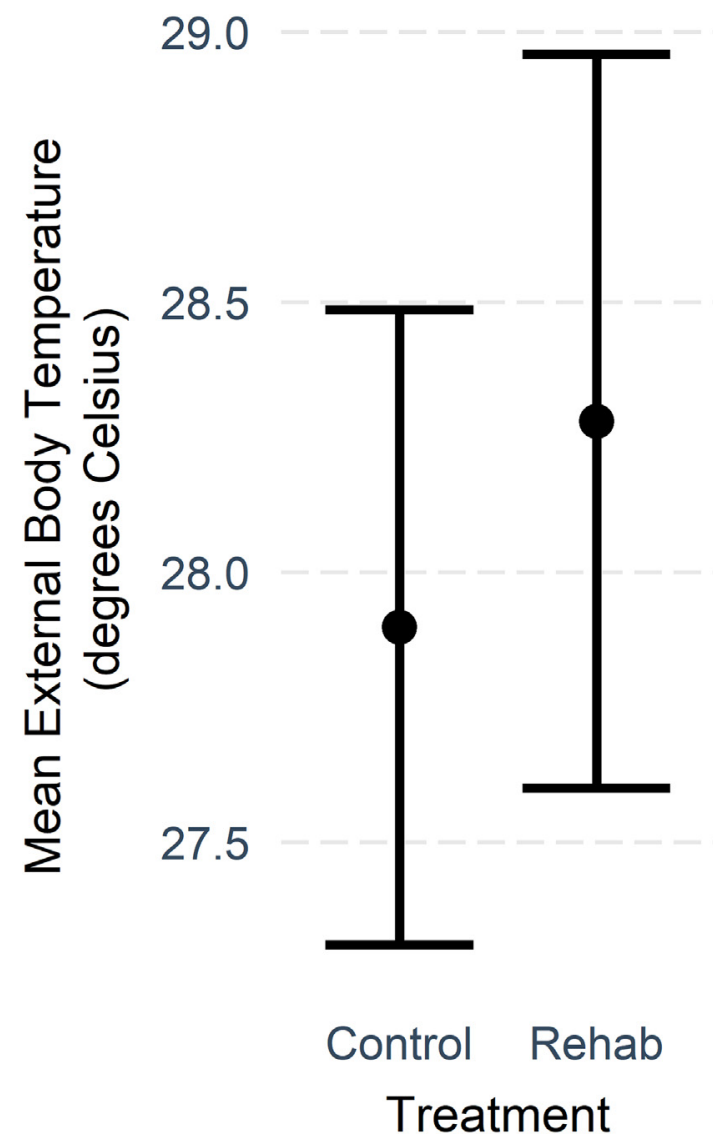

Fig. 7. Marginal effects plot showing mean external temperatures for Control and Rehabilitated birds based on thermography images taken on days 15, 22, and 31 days post oiling ( 6 or 7,13 or 14 , and 22 or 23 days post cleaning). On average, external temperatures were slightly higher in Rehabilitated gulls compared to Controls birds, but were not significantly different.
Writing - review \& editing. Emma M. Campbell: Methodology, Investigation, Writing - review \& editing. Nicholas G. Dannemiller: Conceptualization, Methodology, Investigation, Writing - review \& editing. Susan A. Shriner: Conceptualization, Methodology, Investigation, Formal analysis, Resources, Data curation, Writing - original draft, Supervision, Project administration, Funding acquisition.

\section{Declaration of competing interest}

The authors declare that the research was conducted in the absence of any commercial or financial relationships that could be construed as a potential conflict of interest.

\section{Acknowledgments}

The authors would like to thank Lisa Wolfe, Kevin Bentler, Katherine Dirsmith, and Mikaela Samsel for their assistance with data collection. The authors would also like to thank the animal care staff of the National Wildlife Research Center for their dedicated animal husbandry.

\section{References}

Bates, D., Maechler, M., Bolker, B., Walker, S., 2015. Fitting linear mixed-effects models using lme4. J. Stat. Softw. 67, 1-48. https://doi.org/10.18637/jss.v067.i01.

Bigger, S.W., Ngeh, L N . Dann, P., Orbell, J.D., 2017. Towards a quantitative indicator of feather disruption following the cleansing of oiled birds. Mar. Pollut. Bull. 120, 268-273. https://doi.org/10.1016/j.marpolbul.2017.04.051.

Birkhead, T., Lloyd, C., Corkhill, P., 1973. Oiled seabirds successfully cleaning their plumage. British Birds 66, 535-537.

Camphuysen, K.C., 2011. Seabirds and chronic oil pollution: self-cleaning properties of gulls, Laridae, as revealed from colour-ring sightings. Mar. Pollut. Bull. 62, 514-519. https://doi.org/10.1016/j.marpolbul.2010.12.008.

Clark, R.B., 2001. Marine Pollution. Oxford University Press, New York, NY,

Cunningham, F., Dean, K., Hanson-Dorr, K., Harr, K., Healy, K., Horak, K., et al., 2017. Development of methods for avian oil toxicity studies using the double crested cormorant (Phalacrocorax auritus). Ecotoxicol. Environ. Saf. 141, 199-208. https://doi.org/ 10.1016/j.ecoenv.2017.03.025.

Dannemiller, N.G., Horak, K.E., Ellis, J.W., Barrett, N.L., Wolfe, L.L., Shriner, S.A., 2019. Effects of external oiling and rehabilitation on hematological, biochemical, and blood 
gas analytes in ring-billed gulls (Larus delawarensis). Frontiers in Veterinary Science https://doi.org/10.3389/fvets.2019.00405.

De La Cruz, S.E.W., Takekawa, J.Y., Spragens, K.A., Yee, J., Golightly, R.T., Massey, G., et al., 2013. Post-release survival of surf scoters following an oil spill: an experimental approach to evaluating rehabilitation success. Mar. Pollut. Bull. 67, 100-106. https://doi. org/10.1016/j.marpolbul.2012.11.027.

Dixon, T.J., Dixon, T.R., 1976. Olympic Alliance oil spillage. Mar. Pollut. Bull. 7, 86-90. https://doi.org/10.1016/0025-326X(76)90147-8.

Dorr, B.S., Mathewson, P.D., Hanson-Dorr, K.C., Healy, K.A., Horak, K.E., Porter, W., 2020. Landscape scale thermoregulatory costs from sublethal exposure to Deep Water Horizon oil in the double-crested cormorant. Mar. Pollut. Bull. 152, 110915. https://doi. org/10.1016/j.marpolbul.2020.110915.

Miller, D.S., Peakall, D.B., Kinter, W.B., 1978. Ingestion of crude oil: sublethal effect in herring gull chicks. Science. 199, pp. 315-317.. https://doi.org/10.1126/science.145655.

Duerr, R.S., 2013. Investigation into nutritional condition and digestive capabilities of seabirds during rehabilitation in California. Wildlife Management; Zoology; Veterinary Medicine. Ph.D. University of California, Davis, p. 128.

Estes, J.A., 1998. Concerns about rehabilitation of oiled wildlife*. Conserv. Biol. 12, 1156-1157. https://doi.org/10.1046/j.1523-1739.1998.97507.x.

Finlayson, G.R., Louise Chilvers, B., Pearson, H., White, B.J., Finlayson, S.T., Sievwright, K., et al., 2018. Efficacy of seawater for washing oiled birds during an oil spill response. Mar. Pollut. Bull. 126. https://doi.org/10.1016/j.marpolbul.2017.10.080.

Fritt-Rasmussen, J., Linnebjerg, J.F., Sorensen, M.X., Brogaard, N.L., Riget, F.F., Kristensen, P., et al., 2016. Effects of oil and oil burn residues on seabird feathers. Mar. Pollut. Bull. 109, 446-452. https://doi.org/10.1016/j.marpolbul.2016.05.029.

Goldsworthy, S.D., Gales, R., Giese, M., Brothers, N., 2000a. Effects of the Iron Baron Oil Spill on Little Penguins (Eudyptula minor). I. Estimates of Mortality. 27. CSIRO Wildlife Research, pp. 559-571.. https://doi.org/10.1071/wr99075.

Goldsworthy, S.D., Giese, M., Gales, R., Brothers, N., Hamill, J., 2000b. Effects of the Iron Baron Oil Spill on Little Penguins (Eudyptula minor). II. Post-Release Survival of Rehabilitated Oiled Birds. 27. CSIRO Wildlife Research, pp. 573-582.. https://doi.org/ 10.1071/wr99076.

Golightly, R.T., Gabriel, P.O., lockerby, cL, Cruz, S.E.W., Takekawa, J.Y., Henkel, L.A., et al., 2019. Post-release behavior of surf scoters (Melanitta perspicillata) following an oil spill: an experimental approach to evaluating rehabilitation success. Waterbirds 39-50 (12), 42. https://doi.org/10.1675/063.042.0105.

Haney, J.C., Geiger, H.J., Short, J.W., 2014. Bird mortality from the Deepwater Horizon oil spill. I. Exposure probability in the offshore Gulf of Mexico. Mar. Ecol. Prog. Ser. 513, 225-237.

Hartung, R., 1967. Energy metabolism in oil-covered ducks. J. Wildl. Manag. 31, 798. https://doi.org/10.2307/3797987.

Henkel, L.A., Ziccardi, M.H., 2018. Life and death: how should we respond to oiled wildlife? J. Fish Wildl. Manag. 9, 296-301. https://doi.org/10.3996/062017-jfwm-054.

Horak, K.E., Bursian, S.J., Ellis, C.K., Dan, K.M., Link, J.E., Hanson-Dorr, K.C., Cumming, F.L., Harr, K.E., Prtisos, C.A., 2017. Toxic effects of orally ingested oil from the Deepwater Horison spill on laughing gulls. Ecotoxicology and Enviromental Safety 146, 83-90.

Jenssen, B.M., 1994. Review article: effects of oil pollution, chemically treated oil, and cleaning on thermal balance of birds. Environ. Pollut. 86, 207-215. https://doi.org/ 10.1016/0269-7491(94)90192-9.

Jenssen, B.M., Ekker, M., 1988. A method for evaluating the cleaning of oiled seabirds. Wildl. Soc. Bull. (1973-2006) 16, 213-215. https://www.jstor.org/stable/3782193.

Jessup, D.A., 1998. Rehabilitation of oiled wildlife. Conserv. Biol. 12, 1153-1155. https:// doi.org/10.1046/j.1523-1739.1998.97522.x.

Jessup, D.A., 2010. Oiled Wildlife Infrared Camera SSEP Study P0475035 (PDF). California Department of Fish and Game, Office of Spill Prevention and Response (Accessed 10/ 15/2019).

Lambert, G., Peakall, D.B., Philogene, B.J., Engelhardt, F.R., 1982. Effect of oil and oil dispersant mixtures on the basal metabolic rate of ducks. Bull. Environ. Contam. Toxicol. 29, 520-524. https://doi.org/10.1007/bf01669614.

Leighton, F.A., 1993. The toxicity of petroleum oils to birds. Environ. Rev. 1, 92-103. https://doi.org/10.1139/a93-008.

Long, J.A., 2019. jtools: Analysis and Presentation of Social Scientific Data.
Maggini, I., Kennedy, L.V., Macmillan, A., Elliott, K.H., Dean, K., Guglielmo, C.G., 2017. Light oiling of feathers increases flight energy expenditure in a migratory shorebird. J. Exp. Biol. 220, 2372-2379. https://doi.org/10.1242/jeb.158220.

Matcott, J., Baylis, S., Clarke, R.H., 2019. The influence of petroleum oil films on the feather structure of tropical and temperate seabird species. Mar. Pollut. Bull. 138, 135-144. https://doi.org/10.1016/j.marpolbul.2018.11.010.

Mathewson, P.D., Hanson-Dorr, K.C., Porter, W.P., Bursian, S.J., Dean, K.M., Healy, K., et al., 2018. Experimental and modeled thermoregulatory costs of repeated sublethal oil exposure in the Double-crested Cormorant, Phalacrocorax auritus. Mar. Pollut. Bull. 135, 216-223. https://doi.org/10.1016/j.marpolbul.2018.07.003.

McCafferty, D.J., 2007. The value of infrared thermography for research on mammals: previous applications and future directions. Mammal Rev. 37, 207-223. https://doi.org/ 10.1111/j.1365-2907.2007.00111.x.

Morandin, L.A., O'Hara, P.D., 2014. Fish oil disrupts seabird feather microstructure and waterproofing. Sci. Total Environ. 496, 257-263. https://doi.org/10.1016/j. scitotenv.2014.07.025.

Munilla, I., Arcos, J.M., Oro, D., Álvarez, D., Leyenda, P.M., Velando, A., 2011. Mass mortality of seabirds in the aftermath of the Prestige oil spill. Ecosphere 2,1-14. https://doi. org/10.1890/ES11-00020.1.

Nevins, H.M., Young, C., Jessup, D.A., 2012. OWN 2010-2011 Final Report: Validation of Thermography to Assess Body Temperature and Waterproofing of Captive and Rehabilitated Seabirds. Oiled Wildlife Care Network (Accessed 10/15/2019, cited with consent of author)

Newman, S.H., Golightly, R.T., Craig, E.N., Carter, H.R., Kreuder, C., 2004. The Effects of Petroleum Exposure and Rehabilitation on Post-Release Survival, Behavior, and Blood Health Indices: A Common Murre (Uria aalge) Case Study Following the Stuyvesant Petroleum Spill. Final Report. Oiled Wildlife Care Network, Wildlife Health Center, UC Davis, CA.

O'Hara, P.D. Morandin, LA.,2010. Effects of sheens associated with offshore oil and gas development on the feather microstructure of pelagic seabirds. Mar. Pollut. Bull. 60 672-678. https://doi.org/10.1016/j.marpolbul.2009.12.008.

Perez, C.R., Moye, J.K., Cacela, D., Dean, K.M., Pritsos, C.A., 2017. Low level exposure to crude oil impacts avian flight performance: the Deepwater horizon oil spill effect on migratory birds. Ecotoxicol. Environ. Saf. 146, 98-103. https://doi.org/10.1016/j. ecoenv.2017.05.028.

Phillips, J., 1974. Oiled seabirds successfully cleaning their plumage. British Birds 67, 483.

R Core Team, 2019. R: A Language and Environment for Statistical Computing. R Foundation for Statistical Computing, Vienna, Austria.

Rijke, A.M., Jesser, W.A., 2011. The water penetration and repellency of feathers revisited Condor 113, 245-254. https://doi.org/10.1525/cond.2011.100113.

Romero, A.F., Oliveira, M., Abessa, D.M.S., 2018. A simple bird sensitivity to oil index as a management tool in coastal and marine areas subject to oil spills when few biological information is available. Mar. Pollut. Bull. 128, 460-465. https://doi.org/10.1016/j. marpolbul.2017.12.008.

Sharp, B.E., 1996. Post-release survival of oiled, cleaned seabirds in North America. Ibis 138, 222-228. https://doi.org/10.1111/j.1474-919X.1996.tb04332.x.

Srinivasan, S., Chhatre, S.S., Guardado, J.O., Park, K.-C., Parker, A.R., Rubner, M.F., et al., 2014. Quantification of feather structure, wettability and resistance to liquid penetration. J. R. Soc. Interface 11, 20140287. https://doi.org/10.1098/rsif.2014.0287.

Trustee Shorebird Technical Work Group, 2010. Work plan for estimating shorebird oiling and mortality Deepwater Horizon (Mississippi Canyon 252) oil spill bird study. , p. 21 Accessed on. https://www.gulfspillrestoration.noaa.gov/sites/default/files/wp-content/uploads/BirdStudy_5_NonbreedingShorebirds_Final_-Executed-Plan-2_ Redacted.pdf, Accessed date: February 2020.

Whitmer, E.R., Elias, B.A., Harvey, D.J., Ziccardi, M.H., 2018. An experimental study of the effects of chemically dispersed oil on feather structure and waterproofing in common Murres (Uria aalge). J. Wildl. Dis. 54, 315-328, https://doi.org/10.7589/2017-01-016.

Wickham, H., 2016. ggplot2: Elegant Graphics for Data Analysis. Springer-Verlag, New York.

Wilke, C.O., 2020. ggridges: ridgeline plots in 'ggplot2'. R package version 0.5.2. https:// CRAN.R-project.org/package $=$ ggridges. 